\begin{tabular}{lc}
\hline & ANNALES \\
& UNIVERSITATIS MARIAE CURIE-SKŁODOWSKA \\
LOL. V & SECTIO N \\
\hline
\end{tabular}

ISSN: 2451-0491 • e-ISSN: 2543-9340 - CC-BY 4.0 • DOI: 10.17951/en.2020.5.473-486

\title{
Kulturbedingte Symbolkraft von Farben am Beispiel von Gelb
}

\author{
The Cultural Symbolism of Colors Using the Example of Yellow
}

Kulturowa symbolika kolorów na przykładzie koloru żółtego

\author{
Patrizio Malloggi \\ Universität von Pisa. Institut für Philologie, Literatur und Linguistik \\ Piazza Evangelista Torricelli, 2, 56126 Pisa PI, Italien \\ patrizio.malloggi@fileli.unipi.it \\ https://orcid.org/0000-0001-8026-2470
}

\begin{abstract}
Colour terms behave quite differently across languages, similar to the way in which our psychological interpretation varies in response to reflected visible light. Moving from this general overview to a more precise analysis of the colour terms, this paper investigates the concrete as well as symbolic meaning of the colour yellow in the German language at different stages in the development of this language, from the medieval up to the present day. Furthermore, this paper focuses on the metaphorical uses of the colour yellow, which share the same meanings with other European languages as well as a specific meaning characterizing the German culture. The different meanings and emotional values of the colour yellow are investigated in a corpus from the database of the Institut für Deutsche Sprache (Mannheim). The aim of the present paper is to show that the colour yellow is characterized by intercultural as well as by specific meanings related to the German culture.
\end{abstract}

Keywords: symbolic meaning of the colour yellow; analysis of the colour terms; emotional values of the colour yellow 


\begin{abstract}
Abstrakt. Nazwy określające kolor są zupełnie inne w różnych językach, podobnie jak nasza psychologiczna interpretacja zmieniają się w odpowiedzi na odbite światło. Przechodząc od tej ogólnej panoramy do dokładniejszej analizy nazw kolorów, w niniejszym artykule zbadano konkretne i symboliczne znaczenie koloru żółtego w języku niemieckim na różnych etapach rozwoju tego języka: od średniowiecza po współczesność. Ponadto skupiono się na metaforycznych zastosowaniach koloru żółtego, które mają te same znaczenia w innych językach europejskich, a także specyficzne znaczenie charakteryzujące kulturę niemiecką. Różne znaczenia i wartości emocjonalne koloru żółtego są badane w korpusie z bazy danych Institut für Deutsche Sprache (Mannheim). Celem artykułu jest wykazanie, że kolor żółty charakteryzuje się międzykulturowymi i specyficznymi znaczeniami związanymi z kulturą niemiecką.
\end{abstract}

Słowa kluczowe: symboliczne znaczenie koloru żółtego; analiza nazw kolorów; wartości emocjonalne koloru żółtego

\begin{abstract}
Abstrakt. Farbbegriffe verhalten sich je nach Sprache recht unterschiedlich, ähnlich wie unsere psychologische Interpretation als Reaktion auf reflektiertes sichtbares Licht variiert. Von diesem allgemeinen Hintergrund zu einer genaueren Analyse der Farbbegriffe übergehend, untersucht die vorliegende Arbeit die konkrete wie symbolische Bedeutung der Farbe Gelb in der deutschen Sprache in verschiedenen Entwicklungsstadien dieser Sprache, vom Mittelalter bis zur Gegenwart. Darüber hinaus konzentriert sich dieser Beitrag auf die metaphorischen Verwendungen der Farbe Gelb, die mit anderen europäischen Sprachen die gleichen Bedeutungen teilen, sowie auf eine spezifische Bedeutung, die die deutsche Kultur charakterisiert. Die verschiedenen Bedeutungen und emotionalen Werte der Farbe Gelb werden in einem Korpus aus der Datenbank des Instituts für Deutsche Sprache (Mannheim) untersucht. Ziel der vorliegenden Arbeit ist es zu zeigen, dass die Farbe Gelb sowohl durch interkulturelle als auch durch spezifische, die deutsche Kultur kennzeichnende Bedeutungen gekennzeichnet ist.
\end{abstract}

Schlüsselwörter: symbolische Bedeutung der Farbe Gelb; Analyse der Farbbegriffe; emotionale Werte der Farbe Gelb

\title{
EINFÜHRUNG
}

Farben sind seit mehr als 2500 Jahren ein bevorzugtes Forschungsobjekt, an dem man exemplarisch versucht, Aufschluss über die Beziehung zwischen Physikalischem und Psychologischem sowie zwischen dem Objektiven und dem Subjektiven zu erhalten. Demokrit, Descartes, Hume, Locke, Kant oder Russell, um nur einige bekannte Namen zu nennen, haben sich ebenso mit der Natur der Farben beschäftigt wie Galilei, Newton, oder Maxwell. Auch in der Kognitionsforschung stellen die Farben einen in seiner Reichhaltigkeit einzigartigen Mikrokosmos dar, in dem sich Physik, Wahrnehmungsforschung, Neurophysiologie, Evolutionsbiologie, Entwicklungspsychologie und Linguistik miteinander verbinden (Mausfeld 2007: S. 1-2). Der sprachwissenschaftliche Ansatz zeichnet sich dadurch aus, dass er nicht an der physikalischen Farbwahrnehmung, sondern vielmehr an der inneren psychischen 
Farbwahrnehmung interessiert ist. Die innere Farbwahrnehmung ist eine psychische Reaktion auf die wahrgenommene Farbe. Die Farbe als solche wird entmaterialisiert und wird zu einer inneren Erfahrung, wobei die hervorgerufene Emotion vordergründig wird. Farbe bewegt jedoch auch unseren Geist und weckt Assoziationen zu bereits gemachten Farberfahrungen. Dazu kommen tausendjährige kulturelle und symbolische Farb-Bedeutungen, die im Unterbewusstsein schlummern. Nach dem sprachwissenschaftlichen Ansatz werden Farben symbolische Bedeutungen zugeschrieben, die das Ergebnis ihrer Überarbeitung einer bestimmten Kultur in einer bestimmten Epoche sind (Berlin und Kay 1969; Grossmann 1988; Leonardi 2000: S. 47).

Ähnlich wie den Tieren werden auch den Farben seit jeher symbolische Bedeutungen zugeschrieben. Farbensymbolik spielt z.B. in der Kunst, im Märchen, in der Psychologie eine bedeutsame Rolle. Farben werden im Volksglauben, in der Malerei und in religiösen Bereichen als Kultursymbole betrachtet und dienen in verschiedenen Kulturen als Ordnungsprinzipien der Welt. Verbreitet war die Zuordnung von Farben z.B. im altchinesischen und mittelalterlich-abendländischen Denken zu den Himmelsrichtungen, zu den Planeten, Jahreszeiten, Temperamenten (Dobrovol'skij und Piirainen 1997: S. 227-228).

Der vorliegende Beitrag beruht auf der semantischen Untersuchung des Farbadjektivs gelb, d.h. auf seiner Bedeutung bzw. unterschiedlichen Verwendungsweisen; dabei wird der Symbolik der Farbe Gelb besondere Beachtung geschenkt. Hierzu wird eine kulturgeschichtliche Darstellung der Gelbsymbolik vermittelt, die den Zeitraum vom Mittelalter bis zur Gegenwart berücksichtigt. Vorrangiges Ziel dieses Beitrags ist es zu zeigen, dass Gelb zum einen eine leuchtende Farbe mit deutlich positiven Konnotationen ist (es ist nämlich die Farbe der Sonne und des Lichtes, es steht der Symbolbedeutung des Goldes nahe); zum anderen ist Gelb für viele Kulturgemeinschaften, etwa für die deutsche und die italienische, die Farbe von Neid und Eifersucht, Warnung und Gefahr (ibidem: S. 286-287). Diese zwiespältige Bedeutung der Farbe Gelb ist historisch seit dem Mittelalter und in vielen Kulturen bezeugt und hängt einerseits von der Kulturgemeinschaft ab, andererseits wird die Farbwirkung von der Qualität des Gelbs beeinflusst.

Dieser Artikel setzt sich aus fünf Abschnitten zusammen: in Abschnitt 1 werden die Untersuchungsmethode erklärt und die Datengrundlage beschrieben. In diesem Abschnitt wird ferner die diesem Artikel zugrunde liegende Fragestellung formuliert. Abschnitt 2 ist mit der korpusbasierten Untersuchung der Semantik der Farbe Gelb der Kernabschnitt des Artikels. In diesem Abschnitt wird ein Überblick über die erzielten Korpusergebnisse gegeben. Hier werden die Ergebnisse der Untersuchung in Diagrammen aufbereitet und zu einer 
Gesamtschau zusammengeführt. Abschnitt 3 ist der diachronischen und kulturell geprägten Darstellung zur Symbolik der Farbe Gelb gewidmet. In Abschnitt 4 wird die Gelbsymbolik in Phraseologismen thematisiert. In Abschnitt 5 wird das Fazit gezogen.

\section{DATENGRUNDLAGE, FRAGESTELLUNG UND UNTERSUCHUNGSMETHODE}

Wie bereits oben erwähnt, liegt der Schwerpunkt des Forschungsinteresses in dem vorliegenden Artikel nicht auf dem kognitiven Aspekt, sondern auf der sprachlichen Ebene zu Farben; es geht also weniger um die Wahrnehmung der Farben an sich als um die Bedeutungen der einzelnen Farbwörter. Dabei soll das Farbadjektiv gelb primär im Hinblick auf seinen aktuellen Gebrauch untersucht werden. Zu diesem Zweck wurde ein Textkorpus zusammengestellt, das die Untersuchung von authentischem Sprachgebrauch ermöglichen soll und das möglichst aussagekräftige Belege zu den Verwendungsweisen von der Farbe Gelb in relevanten Bereichen des Sprachgebrauchs liefert.

Als Datenbasis dient eine Sammlung von 200 Textausschnitten, die durch das Portal COSMAS ${ }^{1}$ des Instituts für Deutsche Sprache Mannheim zusammengestellt wurde. Die Ausschnitte stammen in der Hauptsache aus Zeitungstexten. Hinzu kommen literarische und populärwissenschaftliche Texte. Die korpusgestützte Untersuchung dient vor allem der Darstellung der Bedeutung der Farbe Gelb in der zeitgenössischen (deutschsprachigen) Kulturgemeinschaft. Hinzu kommen lexikographische Quellen, die einem diachronischen kulturgeschichtlichen Überblick über die unterschiedlichen Verwendungsweisen der Farbe Gelb dienen. Hierdurch kann die Bandbreite der erfassten gelb-Verwendungsweisen erweitert werden.

Der Kontext, in dem der gelb-Satz eingebettet ist, wird für die semantische Interpretation des Farbadjektivs gelb genutzt. Im Fokus der Untersuchung steht durchweg der Satz mit dem gelb-Vorkommen. Der Bezugsausdruck, auf den die Farbe Gelb verweist, wird semantisch klassifiziert.

Aus der Untersuchung der Vorkommen des Farbadjektivs gelb im IDSKorpus wird die zwiespältige Bedeutung dieser Farbe bestätigt, die, wie bereits angedeutet, positive sowie negative Konnotationen hat. Davon geht die Frage aus, ob die festgestellte widersprüchliche Bedeutung der Farbe Gelb typisch für den heutigen Sprachgebrauch oder ob sie auch in früheren sprachgeschichtlichen Verwendungen dieser Farbe nachzuweisen ist.

1 Das Portal COSMAS II ist unter der folgenden Adresse abrufbar: https://cosmas2.ids-mannheim.de/cosmas2-web. 


\section{KORPUSGESTÜTZTE UNTERSUCHUNG DER VERWENDUNGSWEISEN DES FARBADJEKTIVS GELB}

Dieser Abschnitt ist der Präsentation der Korpusergebnisse, auch durch Diagramme, gewidmet.

\section{Korpuserstellung}

Für den vorliegenden Artikel wurde auf die über COSMAS zugänglichen Textkorpora des Instituts für Deutsche Sprache in Mannheim zurückgegriffen: Hier kann man dank einer auf sprachwissenschaftliche Zwecke abgestimmten Suchfunktion Belegstellen aus unterschiedlichsten Quellen schnell und effektiv sammeln. Die Grundlage des entsprechenden Korpus bilden vor allem Zeitungstexte, die angesichts der verschiedenen Bereiche (Politik, Kultur, Sport usw.) zumindest eine gewisse Vielfalt an möglichen Verwendungskontexten des Farbadjektivs gelb bieten.

\section{Korpusergebnisse}

Aus der Untersuchung der einzelnen Vorkommen des Farbadjektivs gelb im Korpus ergibt sich, dass die Farbe Gelb zum einen eine konkrete, zum anderen eine übertragene Verwendung aufweist. Die unten stehende Grafik 1 zeigt die Verteilung der Gebrauchsweisen der Farbe Gelb im Korpus.

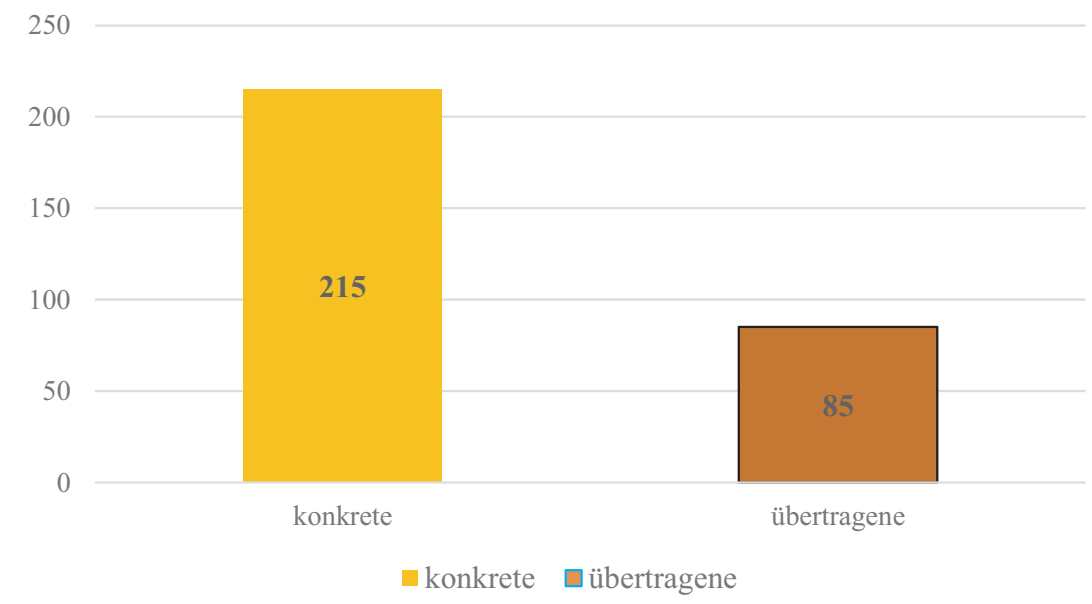

Grafik 1. Verwendungen des Farbadjektivs gelb 
Unter konkreter Verwendung des Farbadjektivs gelb ist gemeint, dass sie auf natürliche bzw. künstliche Farbträger verweist. Zu den natürlichen Farbträgern zählt man die Kategorie der Blumen (Sonnen-, Dotterblumen) und der Früchte (reife Zitronen, Bananen), die innerhalb der Gruppe der natürlichen Farbträger mit Abstand die größte Teilmenge darstellen. Hinzu kommen die Kategorie der Tiere (Bienen, Kanarienvögel), der Gemüsesorten (Kürbisse, Rüben) und der Nahrungsmittel (Eidotter). Gelb ist auch die Farbe von Naturerscheinungen wie der Sonne. Den künstlichen Farbträgern gehören Kleidungsstücke (Hemden, Jacken, usw.), Verkehrszeichen (Ampel), Chemikalien (Schwefel) und sonstige Gegenstände, denen der Mensch die Farbe Gelb zu bestimmten Zwecken zugeschrieben hat (Abfallgebührenmarken). Grafik 2 veranschaulicht die Verteilung der oben erwähnten Kategorien im Korpus.

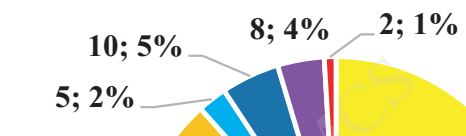

$15 ; 7 \%$

80; $37 \%$

\section{$30 ; 14 \%$}

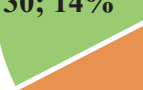

$65 ; 30 \%$

\begin{tabular}{|c|c|c|}
\hline Blumen & - Früchte & - Tiere \\
\hline - Nahrungsmittel & - Kleidun & - Verke \\
\hline
\end{tabular}

Grafik 2. Verteilung der Gelbkategorien im Korpus

Grafik 3 verdeutlicht die Verteilung der Korpusvorkommen, in denen das Farbadjektiv gelb zur Bezeichnung von Elementen des Naturreichs dient. 


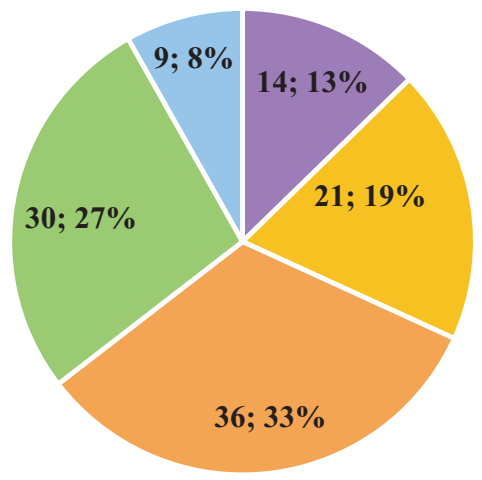

- Blumen $\quad$ Früchte $\quad$ Tiere $\quad$ Gemüsesorten $\quad$ Sonne, Sand

Grafik 3. Verteilung der Gelbvorkommen, die auf das Naturreich verweisen

Die übertragene Verwendung der Farbe Gelb verweist auf die symbolische Bedeutung, die das Gelb im Verlauf der Zeit übernommen hat. In seiner symbolischen Bedeutung gilt das Gelb als Abzeichen von Neid, Notfall, Krankheit und Verwarnung, wie Grafik 4 deutlich zeigt.

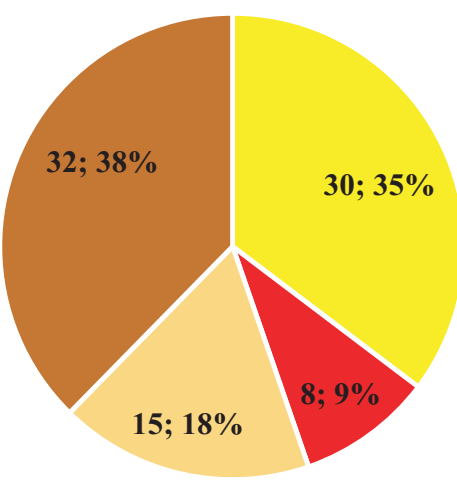

- Neid - Gefahr $\quad$ Krankheit - Verwarnung

Grafik 4. Verteilung der Gelbsymbolik im Korpus

In einigen europäischen Sprachen, wie dem Deutschen oder dem Italienischen, zeigt sich die Assoziation von Gelb mit Neid, was mit kulturell und volkstümlich verankerten Vorstellungen, beispielsweise mit der Säftelehre, übereinstimmt: 
(1) Diese Frauen sind wirklich zu beneiden (bloß gut, dass Sie mich beim Schreiben dieser Zeilen nicht sehen können, denn ich bin schon gelb vor Neid! (Korpusbeleg)

Nach der Volksmeinung färbt sich das Gesicht von neidischen (und haßerfüllten) Menschen durch die Wirkung der Galle gelb; die Galle galt als Sitz von Ärger und Neid (Dobrovol'skij und Piirainen 1997: S. 287).

Auffällig ist Gelb als Gefahrensymbol, das sich insbesondere infolge der Industrialisierung (des Kernkraftwerks) durchgesetzt hat: für radioaktive Stoffe verwendet man einen strahlenden Behälter mit Schwarz auf gelbem Grund.

Gelb gilt als Anzeichen von Krankheit und Tod:

(2) Ich seh' die Todten, Sie liegen unten in den schmalen Särgen, Die Händ' gefaltet und die Augen offen, Weiß das Gewand und weiß das Angesicht, Und durch die gelben Lippen kriechen Würmer. (Korpusbeleg)

Das Signalisieren einer gelben Flagge auf einem Schiff bedeutet den Ausbruch der Seuche.

International wird die Farbe Gelb als Warnfarbe verwendet: Gelb-schwarze Streifen müssen beim Blindenabzeichen unbedingt beachtet werden. Im Tierreich gilt Gelb wie Rot als Warnfarbe und signalisiert Gift, so auch beim Feuersalamander oder bei Wespen und Hornissen (Heller 1989).

Im Fußball verwarnt der Schiedsrichter die Spieler bei einem Foul mit einer gelben Karte:

(3) Poll bekam Gelb, weil er seinen Gegenspieler gefoult haben soll. (Korpusbeleg)

Das Augenmerk wird in dem vorliegenden Artikel auf die symbolische Bedeutung der Farbe Gelb gerichtet, die die Ausgangsbasis für die in dem folgenden Abschnitt vermittelte Darstellung über die kulturgeschichtliche Entwicklung der Gelbsymbolik bildet.

Aus der Korpusuntersuchung geht hervor, dass sich mit der Farbe Gelb ${ }^{2}$ verschiedene, zum Teil widersprüchliche Vorstellungen verbinden. Zum einen ist sie die leuchtende Farbe, wie sie in der Natur in Frühlings- und Sommerblumen oder einem reifen Kornfeld vorkommt, mit deutlich positiven Konnotationen; das Gelb ist die Farbe der Sonne und des Lichtes, es steht der Symbolbedeutung des Goldes nahe (siehe auch Paul 1992: 329; Dobrovol'skij und Piirainen 1997:

2 Ableitung vom Althochdeutschen gelo (Grimm 1897: S. 2879 ff.; Pfeifer Hrsg. 1997: S. 417). 
S. 286; Wahrig 2011: S. 121; Jones 2013: S. 1124 ff.). Zum anderen ist Gelb die Farbe des Neids, der Gefahr, Krankheit und Verwarnung.

Die kontextbezogene Analyse der Bedeutung der Farbe Gelb hat gezeigt, dass diese zwiespältige Farbwirkung des Gelbs von kulturellen Unterschieden abhängt; die Farbwirkung wird ferner aber auch von der Qualität des Gelbs beeinflusst: Schon ein leichter Grauschleier lässt Gelb schmutzig erscheinen und ihm negative Konnotationen zuschreiben. Schon im Mittelalter wird das Goldgelb als heilige und göttliche Farbe gesehen, während ein grelles, grünliches Gelb für Gebrandmarkte und Ausgestoßene gebraucht wurde. Die kulturell geprägte Wirkung bzw. Bedeutung der Farbe Gelb wird in Abschnitt 3 thematisiert.

\section{KULTURGESCHICHTLICHE DARSTELLUNG ZUR GELBSYMBOLIK}

Als Anzeichen des Neides ist Gelb im Deutschen schon im frühen 13. Jahrhundert bezeugt; die Verbindung der Farbe Gelb mit Neid kommt beispielsweise in dem folgenden Dialog aus Das narren-schneyden (1557) von Hans Sachs vor:

(1) Der kranck: Mein lieber herr, wer ist der selbl Narr so dürr, mager, blaich und gelb? Der arztet spricht: Schaw! dieser ist der neydig narr. (Wanzeck 2003: S. 75-93)

Der gelbe Neid ist aller Wahrscheinlichkeit nach mit Hilfe der Säftelehre ${ }^{3} \mathrm{zu}$ erklären, die davon ausgeht, dass durch die Säfte das physische und psychische Leben gesteuert wird. In der Säftelehre wird zwischen der schwarzen und der gelben Galle getrennt. Die schwarze Galle weist auf die Melancholie und die gelbe Galle auf die Bösartigkeit hin. In mittelhochdeutscher Zeit ist die Galle zum Schimpfwort für böse und neidische Personen geworden (ibidem: S. 79). Die Galle ist nach ihrer gelb-grünen Farbe benannt worden. Von daher wird die Farbe Gelb in Verbindung mit Neid auf die gelblich-grüne Farbe der Galle zurückzuführen sein. Der Ausdruck der gelbe Neid kommt dann in verschiedenen Formen seit dem 18. Jahrhundert häufiger vor: gelbspritziger Neid (1710), neidisch-gelb (19. Jahrhundert). Nach altem Glauben sitzt der Ärger in der Galle, und wer sich zu viel ärgert, bekommt gelbe Haut, weil die Gallenwege sich verkrampfen und

3 Säftelehre, eine von der Antike bis ins 18. Jahrhundert allgemein anerkannte medizinische Konzeption, die zur Erklärung allgemeiner Körpervorgänge und als Krankheitskonzept entwickelt wurde. Die vier Körpersäfte sind Blut, gelbe Galle, schwarze Galle und Schleim (Eckardt 2017: S. 12 ff.). 
die Galle dann nicht mehr über den Darm abgeführt werden kann. Aus diesem Grund werden noch heute negative Emotionen wie Ärger, Eifersucht, Neid und Egoismus mit der Farbe Gelb in Verbindung gebracht (ibidem: S. 78 ff.).

Ein anderer Überlieferungsstrang der negativen Farbeinschätzung geht auf das Mittelalter zurück, dem zufolge Gelb die Kennfarbe sozialer Stigmatisierung (Deklassierte und Geächtete) war. So verordnete die habsburgische Kleiderordnung des 15. Jahrhunderts den Prostituierten das Tragen eines gelben Kopftuches und ein Leipziger Gesetz von 1506 selbst das Tragen von gelben Umhängen. In anderen Regionen waren gelbe Schleier oder gelber Kleiderbesatz verordnet. In Hamburg mussten „wandelbare Vrouwen“ bereits ab 1445 ein gelbes Kopftuch tragen und in Meran sollten Prostituierte Schuhe mit gelben Bändern tragen. Auch Frauen mit unehelichen Kindern mussten derzeit ihre "Schande" durch gelbe Kleidung offenbaren, so wurde ihnen z.B. in Freiburg im Breisgau das Tragen von gelben Hauben verordnet. Auch Schandkleider, in denen man Hexen folterte und verbrannte, waren gelb. Ketzer, die zum Tode verurteilt waren, mussten auf dem Weg zur Hinrichtung ein gelbes Kreuz tragen. Wer Schulden hatte, musste große gelbe Scheiben auf seine Kleider nähen. Wo Geächtete wohnten, strich man die Türen gelb (Immoos 2009: S. 36; Jones 2013: S. 1124-1138).

In der mittelalterlichen Kunst wird das Kleid des Judas gelb dargestellt. Da Gelb weder in der jüdischen noch in der christlichen Kirche als liturgische Farbe erlaubt ist, eignete es sich gut zur Kennzeichnung der Andersgläubigen. Bereits seit dem 12. Jahrhundert mussten Juden einen auffälligen gelben Hut tragen, der spitz und hoch aufragte, wenn sie ihr Ghetto verließen oder sie waren durch einen gelben Fleck auf der Kleidung gekennzeichnet. Außerdem hatten sie große gelbe Ringe auf ihre Kleider aufzunähen. Nicht allein im Mittelalter, sogar in unserem Jahrhundert, erlebten die Juden Gelb als Farbe der Diskriminierung; die Nationalsozialisten zwangen sie im Dritten Reich, einen gelben Davidsstern zu tragen. In seiner Schrift Zur Farbenlehre (um 1800) stellt Goethe Hypothesen auf, wieso Gelb zur Farbe der Geächteten wird: «wenn die gelbe Farbe unreinen und unedlen Oberflächen mitgeteilt wird, wie dem gemeinen Tuch, dem Filz und dergleichen, worauf sie nicht mit ganzer Energie erscheint, entsteht eine solche unangenehme Wirkung» (Goethe 1810).

Auf das 13. Jahrhundert geht der Bezug des Adjektivs gelb auf Hautfarbe als Anzeichen von Krankheit (vergilben, gelbe Sucht, gelbsüchtig, gelbe Haut) zurück. Ferner wurden psychische Krankheiten wie Wahn, Schizophrenie, Manie und Epilepsie mit dieser Farbe verbunden.

In Europa warnte Gelb bereits seit dem Mittelalter auch vor Gefahr: Wenn in einer Stadt die Pest ausgebrochen war, wurde eine gelbe Flagge gezeigt. Noch 
heute hisst man auf einem Schiff die gelbe Flagge, wenn an Bord eine ansteckende Krankheit ausgebrochen ist. Die Flagge steht im Flaggenalphabet für den Buchstaben Q, was in dem Fall Quarantäne bedeutet (Immoos 2009: S. 37). Der Ausdruck gelbe Gefahr ist eine Lehnübersetzung aus dem Jahr 1900. Zunächst verstand darunter die Immigration der Chinesen seit 1876 nach Amerika. Im Deutschen geht der Ausdruck wahrscheinlich auf den Schriftsteller Stefan von Kotze und dessen Buch Die gelbe Gefahr (um 1900) zurück (Wanzeck 2003: S. 75-93).

Gelb hat schließlich von Alters her auch eine wichtige Bedeutung als Warnfarbe und ist seit 1697 als giftige Farbe bezeugt. Schon im Tierreich hat sich die Farbe Gelb als die beste Warnfarbe mit dem größten Überlebensvorteil bewährt. Sie signalisiert dem Fressfeind, dass die potentielle Beute giftig, gefährlich und ungenießbar ist (Witting 2014: S. 410-412).

\section{GELBSYMBOLIK IN PHRASEOLOGISMEN}

Die Symbolkraft der Farben ist besonders in Phraseologismen ${ }^{4}$ verankert. Die Auswahl der untersuchten historischen und gegenwartssprachlichen Farbphraseologismen erfolgte aus lexikographischen Quellen. Ab 1650 ist eine gewisse idiomatische Produktivität festzustellen (Jones 2013: S. 1127); Gelb kommt beispielsweise in den folgenden Phraseologismen vor: gelber Neid, etwa ein gelbes Brühlein darüber machen, jemandem führt ein Gelbhorn, gelb um den Schnabel, etwas ist (ja) nicht (gerade) das Gelbe vom Ei.

Im Farbphraseologismus gelber Neid dient das Farbadjektiv gelb der Bedeutungsintensivierung. In diesem Phraseologismus beruht die Bedeutungsbeziehung auf natürlichen Farbmerkmalen, indem die Farbe Gelb in Verbindung mit Neid auf die gelblich-grüne Farbe der Galle zurückzuführen ist. Wie oben angedeutet, wird in der Säftelehre zwischen gelber und schwarzer Galle unterschieden, wobei die gelbe Galle auf die Bösartigkeit hinweist.

Im Phraseologismus etwa ein gelbes Brühlein über etwas machen,verleumderische Reden halten' ist gelb in der Bedeutung ,schändlich' motiviert. Der Phraseologismus eine Brühe machen ,Geschwätz, Gerede machen' ist um das Adjektiv gelb ,schmutzig' zu ein gelbes Brühlein über etwas machen ergänzt.

4 Laut Burger (2003: S. 1 ff.) ist eine Aussage phraseologisch, wenn eine Verbindung von zwei oder mehreren Wörtern durch die syntaktischen und semantischen Regularitäten eine nicht völlig erklärbare Einheit bildet, und wenn die Wortverbindung in der Sprachgemeinschaft, ähnlich wie ein Lexem, gebräuchlich ist. 
Die Wendung jemandem führt ein Gelbhorn, jemand (der Mann) wird von seiner Ehefrau betrogen' entspricht der Verbindung Hörner führen, Hahnrei sein'. Das Bestimmungswort gelb in Gelbhorn verweist auf die farbsymbolische Bedeutung der Untreue und des Ehebruchs. Gelb als Kennzeichen der untreuen Ehefrauen ist auf die betrogenen Ehemänner übertragen (Wanzeck 2003: S. 90 ff.).

In der für die norddeutschen Dialekte typischen verbalen Wendung Gelb sprechen bekommt Gelb aufgrund der dieser Farbe zugeschriebenen negativen Farbqualität die symbolische Bedeutung ,schmutzig' (Jones 2013: S. 1136). Das Adjektiv gelb bezeichnet ein fehlerhaftes (schmutziges) Hochdeutsch, so dass gelb sprechen auf Leute bezogen wird, die hochdeutsch sprechen, ohne es recht zu können.

Die Wendung gelb um den Schnabel sein beruht auf einer Bedeutungsübertragung aus dem Tierreich. Dort ist der Schnabelansatz junger Vögel mit einer gelben Haut überzogen. Die pejorativ gebrauchte Bezeichnung Gelbschnabel lässt sich seit dem 16. Jahrhundert nachweisen. Die Redensart gelb um den Schnabel sein bedeutet, jemand verhält sich nicht gemäß seinem Alter, jemand verhält sich dumm' (ibidem).

In der Wendung etwas ist (ja) nicht (gerade) das Gelbe vom Ei ,etwas ist (nicht) das Bestmögliche' trägt Gelb aufgrund der natürlichen Farbmerkmale die positive Bedeutung ,das Beste'. Mit Gelb ist der Eidotter, das Beste am ganzen $\mathrm{Ei}$, gemeint. Bekannt ist auch die Redewendung jemandem gelb und grün vor Augen werden. Diese Redewendung beruht wahrscheinlich auf dem Zustand bei einem eintretenden Schwindelgefühl: Diese Kreislaufschwäche kann diese Farberscheinungen im Auge mit sich bringen. Einen ähnlichen Zustand (Ohnmacht) kann auch gesteigerter Neid auslösen. Jemand, der vor Neid gelb wird, sieht somit kränklich aus.

\section{SCHLUSSBEMERKUNGEN}

Der vorliegende Artikel hat gezeigt, dass Gelb eine sehr zwiespältige Farbe ist. Neben ihren positiven Konnotationen weist die Farbe Gelb eine ganze Reihe negativer Bedeutungen bzw. Verwendungsweisen auf, die kulturell und diachronisch zu erklären bzw. nachzuweisen sind. Die negative Farbwirkung dieser Farbe geht nämlich auf die mittelalterliche Zeit zurück, in der Gelb zur Bezeichnung der sozialen Stigmatisierung (Prostituierte, Ketzer, Frauen mit unehelichen Kindern), der Diskriminierung (Juden), der Krankheit (gelbe Sucht, Pest, Seuche), negativer Gefühle (Neid, Eifersucht) dient. Die meisten dieser negativen Konnotationen werden dem Gelb in unserer europäischen zeitgenössischen Kulturgemeinschaft weiter zugeschrieben und sind ferner auf andere Bereiche übertragen worden, wie auf den Sport-, wo die Farbe Gelb als Verwarnung beim 
Fußballspielen gilt oder auf den Industriebereich (gesundheitsschädliche Stoffe werden durch die Farben Schwarz und Gelb signalisiert). Gelb als Warnfarbe hat sich auch im Tierreich durchgesetzt, in dem es auf Gefahr durch Gift hinweist. Die negative Konnotation der Farbe Gelb erreicht im übertragenen Sinne (in Phraseologismen) ihren Höhepunkt; dies stellt ein weiteres Zeichen für den Zusammenhang zwischen Kultur und Farbbedeutung dar, denn die Denkweise einer bestimmten Kultur kommt in Phraseologismen besonders zum Ausdruck (Burger 2003: S. 101f.). Insofern kann man behaupten, dass die Gelbsymbolik in unserer Kultur eine ausgeprägte negative Farbwirkung hat.

\section{LITERATURVERZEICHNIS}

Berlin, B., Kay, P. (1969). Basic Color Terms: Their Universality and Evolution. Berkeley - Los Angeles: University of California Press.

Burger, H. (2003). Phraseologie: Eine Einführung am Beispiel des Deutschen. Berlin: Schmidt.

COSMAS II. Genommen von: https://cosmas2.ids-mannheim.de/cosmas2-web [Zugang: 10.05.2020].

Dobrovol'skij, D., Piirainen, E. (1997). Symbole in Sprache und Kultur Studien zur Phraseologie aus kultursemiotischer Perspektive. Bochum: Bochum Brockmeyer.

Eckardt, G. (2017). Die griechisch-römische Antike: Von den vier Körpersäften zu den vier Temperamenten (von Hippokrates [4. Jh. vor Chr.] bis Galen [2. Jh. n. Chr.]). In: Persönlichkeits- und Differentielle Psychologie. Wiesbaden: Springer.

Goethe, J.W. von (1810). Zur Farbenlehre. Tübingen.

Grimm, J. und W. (1897). Deutsches Wörterbuch. Leipzig.

Grossmann, M. (1988). Colori e lessico studi sulla struttura semantica degli aggettivi di colore in catalano, castigliano, italiano, romeno, latino ed ungherese. Tübingen: G. Narr.

Heller, E. (1989). Wie Farben wirken. Farbpsychologie, Farbsymbolik, Kreative Farbgestaltung. Reinbek bei Hamburg: Rowohlt Taschenbuch.

Immoos, F. (2009). Farbe. Energie der Farbe. Genommen von: http://franz.immoos.eu/ farbe.pdf [Zugang: 10.05.2020].

Jones, W.J. (2013). Historisches Lexikon deutscher Farbbezeichnungen. Berlin: Akademie-Verlag.

Leonardi, S. (2000). Il colore blu nel tedesco medievale. ata. blâo, atm. Blâ. Annali Sezione Germanica, n. 1, fascicolo X, 47-89.

Mausfeld, R. (2007). Zur Natur der Farbe. Die Organisationsweise von „Farbe“ im Wahrnehmungssystem. In: S. Glasauer, J. Steinbrenner (Hrsg.), Farben. Betrachtungen aus Philosophie und Neurowissenschaften. Frankfurt am Main: Suhrkamp Verlag.

Paul, H. (1992). Deutsches Wörterbuch. Tübingen: Verlag Imprint von de Gruyter.

Pfeifer, W. (Hrsg.). (1997). Etymologisches Wörterbuch des Deutschen. Berlin: Akademie-Verlag. 
Pobrane z czasopisma Annales N - Educatio Nova http://educatio.annales.umcs.pl Data: 26/04/2023 10:26:29

Wahrig. (2011). Deutsches Wörterbuch. Gütersloh: Wissenmedia Verlag.

Wanzeck, C. (2003). Zur Etymologie lexikalisierter Farbwortverbindungen. Untersuchungen anhand der Farben Rot, Gelb, Grün und Blau. Amsterdam - New York: Brill Rodopi.

Witting, W. (2014). Licht. Sehen. Gestalten. Lichttechnische und wahrnehmungspsychologische Grundlagen für Architekten und Lichtdesigner. Basel-Berlin-Boston: Birkhäuser. 